\title{
Workflow Automation Based on OSI Job Transfer and Manipulation
}

\author{
Marten J. van Sinderen ${ }^{\mathrm{a}}$, Stef M.M. Joosten ${ }^{\mathrm{b}}$, and \\ Cléver R. Guareis de Farias ${ }^{a}$

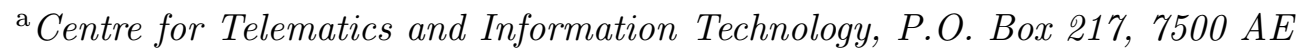 \\ Enschede, The Netherlands \\ ${ }^{\mathrm{b}}$ Anaxagoras Process Architects, Hazenweg 88, 7556 BM Hengelo, The \\ Netherlands
}

\begin{abstract}
This paper shows that Workflow Management Systems (WFMS) and a data communication standard called Job Transfer and Manipulation (JTM) are built on the same concepts, even though different words are used. The paper analyses the correspondence of workflow concepts and JTM concepts. Besides, the correspondence of relationships between those concepts is analysed as well. Thus, we show that JTM is suitable for triggering activities, coordination of work, routing of documents, handling exceptions, safeguarding the integrity of business transactions and retrieving information from business processes. This implies that JTM can be used to support workflows and to provide interoperability between different brands of workflow tools.
\end{abstract}

Key words: interoperability, job transfer and manipulation, network technology, OSI application layer, workflow management systems.

\section{Introduction}

A workflow process is the automated component of a business process [34]. A workflow process consists of activities, and typically crosses boundaries within and between organisations. If a workflow process is orchestrated by different servers of different brands, these servers must be compatible at least on the protocol level. This is a problem within organisations where different departments use workflow servers of different brands. The problem is even more apparent between organisations, because different organisations do not share an authority that might force them into using an interoperable solution. 
Most contemporary workflow products are incompatible, because no standards have yet emerged for terminology, interoperability and connectivity between workflow products. This problem has been recognised by vendors, and has resulted in the foundation of the Workflow Management Coalition (WfMC). The coalition focuses on the workflow management system (abbreviated to WFMS), which is defined as a technological system in which workflow processes are defined, performed, managed and monitored through the execution of software whose order of execution is driven by a computer representation of the workflow process logic [34]. Examples of WFMS include Officetalk-D [8], Action Workflow [25], InConcert [24] and Regatta [32].

Job Transfer and Manipulation (JTM) is an ISO/OSI standard, which can solve the lack of interoperability between different WFMS. Both the JTM standard and WFMS are meant to "orchestrate" business processes, by prompting work, monitoring deadlines, making information available and monitoring the progress of cases in the process. The JTM standard defines a service (ISO 8831) [16] and a protocol (ISO 8832) [17], which provide the full functionality required by WFMSs, allowing organisations to focus their attention on the inherent difficulty of inter-organisational cooperation $[20,33]$.

The remaining of this document is organised as follows. Section 2 outlines our research approach. Section 3 explains the backgrounds of both WFMS and the JTM standard. Section 4 defines workflow and shows which part of a WFMS needs a protocol, the so-called process data. Section 5 introduces the JTM standard. Section 6 shows the conceptual correspondence between the process data and the JTM protocol. Section 7 discusses the future developments in WFMS and the possible impact of JTM. Finally, Section 8 draws some conclusions.

\section{Research Approach}

The main research question, addressed by this paper, is:

Is the JTM standard conceptually suitable for the purpose of automating workflow processes?

In order to answer this question we adopted a conceptual approach to analyse both workflow concepts and JTM concepts. The adoption of a conceptual analysis is sufficient to address this question because it requires no induction in order to draw conclusions of general validity.

This has led to the following steps in the research: (1) define workflow concepts; (2) analyse JTM concepts; (3) relate the concepts of workflow and JTM, and; 
(4) check the correspondence of relationships between concepts.

We have used the results of field studies on workflow management applications [19], the definitions in the Workflow Management Coalition's glossary [34], and papers describing workflow tool architectures $[7,23,25,31]$ to create a conceptual framework of WFMSs (see Section 4). Only part of the framework, i.e., the process data, is related to a data communication service and protocol. The representation of the workflow process logic, which is called definition data, does not interact with the network directly.

The analysis of JTM concepts consists of creating a UML class diagram [5] showing JTM's concepts as object classes and the relationships between those concepts. Being an established and well-defined standard, JTM requires no further justification of its consistency, completeness and applicability. A short and readable introduction to the JTM standard is given in [21], which covers the concepts used in this paper.

Concepts of the workflow process (the process data) and JTM concepts are matched to determine the suitability of JTM for supporting workflow management systems. We decided that the conceptual frameworks of workflow and JTM match if there is a correspondence between the concepts and if there are corresponding relationships between them. Ideally, this relation would be isomorphic. However, some minor differences were found that do not affect the conclusion of our work.

\section{Background}

Office procedure systems and image management systems were the basis of workflow development. Workflow systems originated from office automation $[6,7]$, but only after the inclusion of routing and tracking capabilities in image management systems, the importance of a routing capability for the management of such systems as well as for the management of business processes in general was recognised $[1,14]$. In the following years, some general-purpose workflow management systems were developed, and in the late 80's and early 90's a large number of workflow management products became commercially available. However, the lack of standards for workflow systems made the interoperability between different products a difficult task.

To promote the development of workflow standards the Workflow Management Coalition was created in August 1993. This organisation defined a WFMS a reference model $[13,34]$ that concentrates mainly on interoperability and communication issues. This reference model basically defines the Workflow Enactment Services, a run-time environment consisting of one or more workflow en- 
gines, and five Application Programming Interfaces (APIs) to provide interoperability with these services. Some of the APIs are still in the process of being standardised, but currently a number of products, such as ActionWorks Metro (Action Technologies Inc.), FlowMark (IBM), Staffware (Staffware Corporation) and InConcert (InConcert Inc.), have already been released conforming to the available WfMC standards.

Among the recent trends that have been pointed out recently $[3,10,28,29]$, three are worth mentioning: the adoption of distributed architectures instead of client/server architectures $[4,11,12,14]$, the utilisation of middleware platforms, such as CORBA and WWW, to provide enterprise and inter-enterprise interoperability $[12,14,26,27,30]$, and the integration of workflow systems with the Internet $[2,22,36]$. However, despite all the work that has been carried out by the WfMC, interoperability among existing WFMS is hardly achieved [10].

Two important classes of protocols can be distinguished, viz. application protocols and data transfer protocols. Application protocols are concerned with the interworking aspects of the distributed components of applications, while data transfer protocols are concerned with the quality aspects of data transfer (throughput, delay, reliability), independent of the information contents of the data. For a long time, protocol research has focused mainly on data transfer protocols. The uptake of application protocol research is relatively new, notably since reliable data transfer services with sufficient geographical spans could be provided at acceptable costs, e.g. [9].

JTM is an application protocol standard developed by ISO. Work on JTM initially started with the aim of developing a standard protocol that could replace proprietary remote job entry protocols. The latter protocols enabled one computer to transfer background work to another computer and to receive the results. Soon, however, it was recognised that the work should focus on more general functions for the support of distributed, rather than remote, job processing, for which so far no existing standards were available. The JTM standard that emerged enabled the submission of jobs to one or more other computers, where they can be processed either on-line or off-line; input for the job can be collected at one or more remote locations; output of the job can be returned to the job initiator or delivered at remote locations; and the progression of the job processing can be monitored and manipulated.

The JTM standard was published in 1989, and consists of two parts. One part, ISO 8831 [16], deals with the description of a general model and the definition of the JTM service. The other part, ISO 8832 [17], deals with the specification of the JTM protocol. An overview of the standard can be found in, for example, $[21]$.

Only a few commercial implementations are available to this date, probably 
because the standardisation of JTM took a long time in comparison with other, partially overlapping, standards such as the Distributed Transaction Processing standard. Another possible reason is the lack of specific demands from application areas. Workflow management may become such an application area that can stimulate the uptake of JTM implementations.

Notice that the backgrounds of JTM and WFMS are very distinct in nature. Workflow research until recently neglected interoperability and data communication aspects, and protocol standardisation seemed to be unaware of or too slow to respond adequately to application developments. This might explain why JTM has not been presented as a workflow standard earlier.

\section{Workflow Automation}

Workflow management systems are used to support office procedures, but are also encountered in production. Workflow technology has become feasible because of the widespread availability of data communication services on people's work places. Organisations use WFMSs to monitor cases in a business process, to trigger activities of both human workers and machines, to track ongoing work and to monitor deadlines. Thus, a WFMS is used as an integration tool, primarily for the purpose of integrating business activities into a smoothly running business process. Naturally, this integration requires technical integration of the underlying communication system at the application level.

Figure 1 depicts a generic workflow management system architecture. This architecture shows a distributed system on the basis of a communication network. It is generic in the sense that each of the components can occur zero or more times in different instances. It also abstracts from the concrete machines on which the components are located, i.e., any component can be mapped on any machine. The WFMS itself consists only of those elements that are connected with a filled arrow. The application data and organisational data are part of the complete system, i.e., the set of people, procedures and means within the environment, but not of the WFMS, i.e., the tool.

There are three different types of active components in a WFMS: interface processors, event managers and workflow managers. An interface processor links applications to the WFMS. The event manager maintains a list of work to be done, monitoring deadlines and work conditions and notifying other actors. A workflow manager coordinates within workflow processes, spawns and monitors other workflow processes, and communicates with other workflow managers when necessary. 


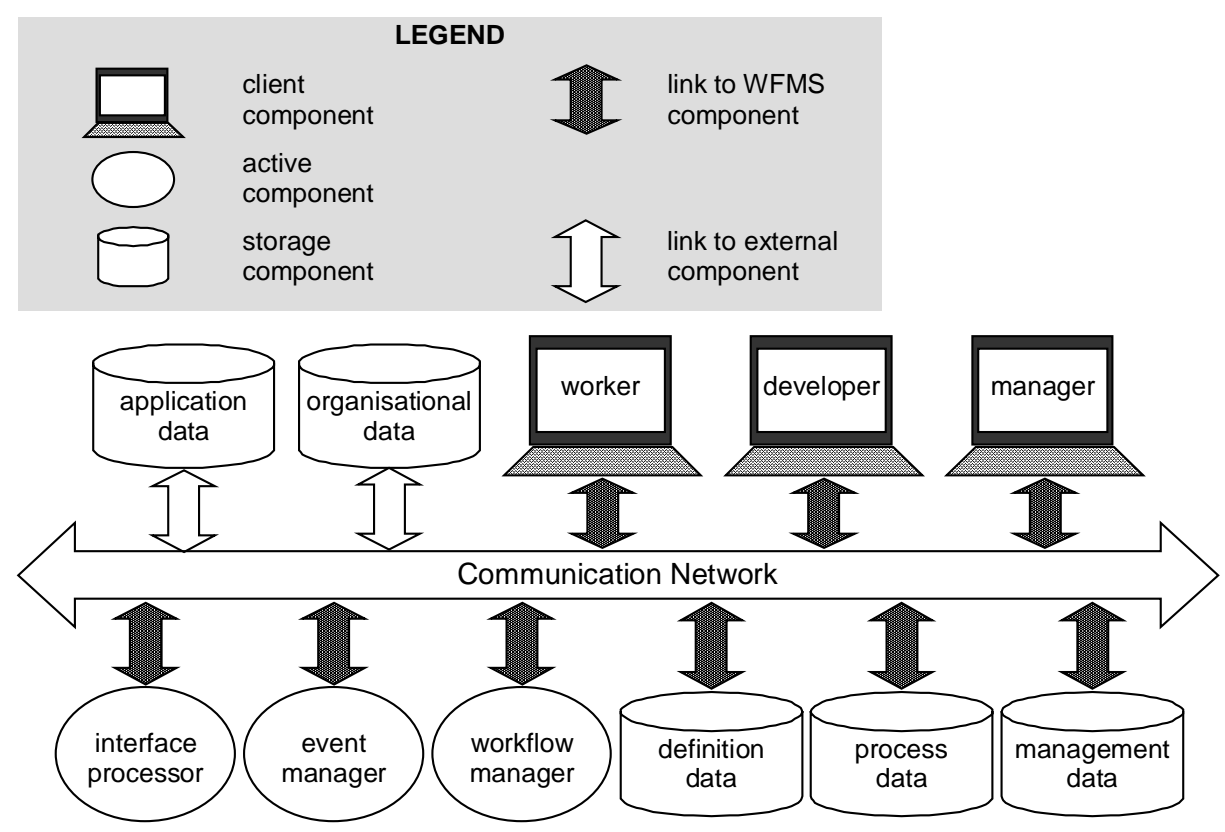

Fig. 1. Generic WFMS architecture.

The clients occur in different roles, such as workers, developers, managers, auditors and quality assurances. These roles are distinguished because each requires a different functionality. In practice, combinations of these roles in one person are not uncommon.

The architecture distinguishes five different kinds of data:

Process data gives information on the current status and history of cases. Process data is used to decide upon the actual order of events, assignment of actors, (re-)scheduling of activities etc. This makes the process data an essential resource for the event managers. Besides, users draw upon the process data in order to track a running workflow process, provide customers with up-to-date status information and knowledge of which activities require attention (the work list, or "to-do" list).

Process Definition data (or simply definition data) gives information on the structure of the workflow processes. It represents the logic of the business process. For workers using the process data, the process definition data contains meta-information. Process definition data can be changed on the fly, thus providing the required flexibility.

Organisation data provides the organisational context of workflow processes. Organisation data is not related to one specific process, but contains information of a more general nature. A WFMW has access to such data in order to respect authorisations and qualifications that are defined within the organisation. Organisation data is not considered to be logically part of the WFMS, but this system has access to the organisation data in order to make decisions that require knowledge about responsibilities and authorisations within business processes. 
Management data gives aggregate information about cases for evaluation purposes. This data is used by (mostly operational) managers to reschedule work when peaks in workloads are anticipated, to obtain insight in operational characteristics of a business process and to obtain metrics for productivity, process times etc.

Application data consists of the documents and data involved in the actual work. Application data is not considered part of the WFMS, but belongs to the applications that are used as part of the business process. The WFMS accesses application data in order to make it available to the persons that perform a specific activity.

The relationship between WFMSs and the JTM standard can be understood in the context of their respective architectures. After the previous discussion of an architecture for workflow management systems, let us discuss the architecture of the JTM standard.

\section{Job Transfer and Manipulation}

\subsection{General concepts}

Like most other OSI standards, the JTM standard consists of a service definition [16] and a protocol specification [17]. The JTM service defines possible interactions between a JTM service provider and JTM service users. A JTM service user is called agency in the standard. Agencies are abstractions of the actual sources and sinks of JTM activity, e.g., printers, file stores, application programs or even human users. The JTM service provider is an abstraction of the distributed system that supports the JTM activity. The JTM service provider consists of JTM-specific components and a lower level service provider. The JTM-specific components, called JTM application service elements (ASEs), interwork via the lower level service provider to provide the JTM service. The rules for interworking are specified by the JTM protocol. Figure 2 illustrates the JTM concepts of service and protocol.

The JTM service provider maintains and manipulates data objects called work specifications. A work specification defines the support expected from the JTM service provider by the agencies. It consists of three key elements:

- a set of global parameters: these parameters enable the identification and authorisation of the entire JTM activity, referred to as the JTM job, and define the report that is required;

- a subjob specification: this element specifies the JTM service provider support for part of the JTM job; 


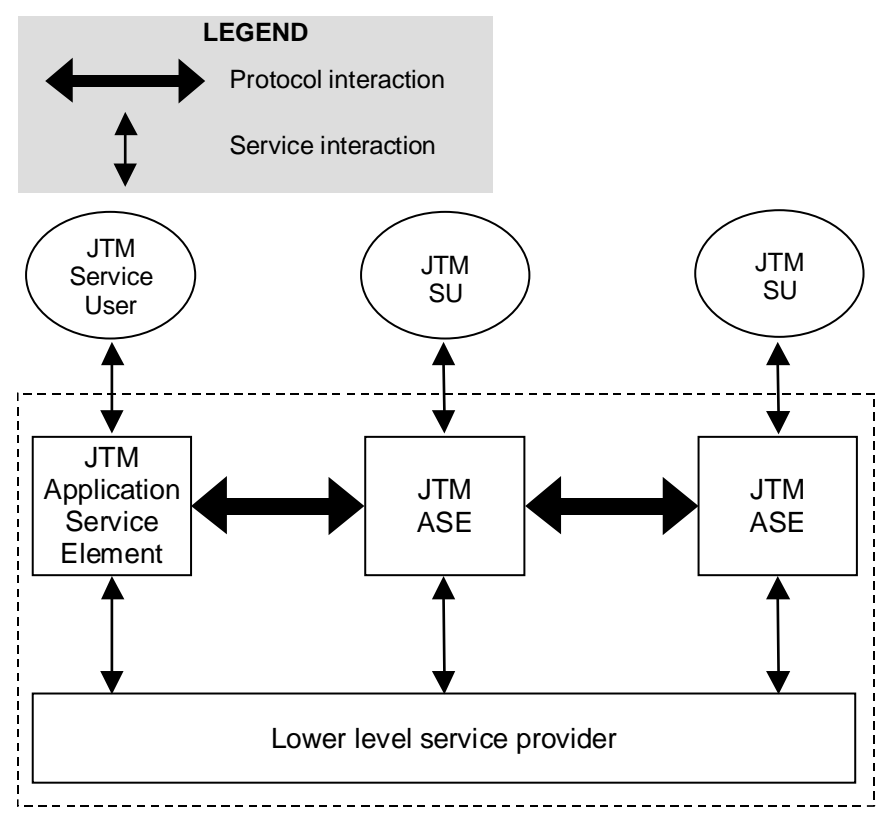

Fig. 2. Concepts of service and protocol.

- a proforma list: this list contains zero or more proformas, each of which contains a subjob specification and a proforma list.

A proforma is a template that is used to create subsequent work specifications through spawning. The concept of proforma allows for conditional and concurrent processing of JTM jobs. After a subjob specified in a work specification has been completed, a new work specification is spawned on the basis of the proforma in that work specification. A request of an agency may also lead to a new work specification, which is called demand spawning. The global parameters apply to the initial subjob as well as to further subjobs obtained by spawning proformas. A subjob coincides with the OSI job if it is the initial subjob and if the proforma list in the initial work specification is empty.

A work specification also contains a document list, which may be empty, with documents which are transparent for the JTM service provider. These documents are required by agencies mentioned in the subjob specification. For example, a document may be a program, a text file, or job data.

Four categories of agencies are distinguished:

- initiation agencies, which are able to define a JTM job. An initiation agency interacts with a JTM ASE, which then creates the initial work specification;

- source agencies, which are able to provide documents to a JTM ASE;

- sink agencies, which are able to accept documents from a JTM ASE;

- execution agencies, which are able to process documents delivered to it by a JTM ASE. An execution agency may also provide documents to the JTM ASE. These documents contain results of the processing of previously 
accepted documents.

Agencies of different types may co-exist in a single (from an OSI point of view, non-distributed) system. Each OSI subjob involves at least a submission system, a single target system, and zero, one or more relay systems. The submission system contains the initiation agency and possibly one or more source agencies; the target system contains execution and/or sink agencies; and relay systems contain source agencies.

\subsection{Document movement}

Document movement is the basic JTM service provider capability for the support of an JTM job. This capability requires a work specification with a document movement subjob type. The document movement subjob defines which documents are to be collected, where they are collected (from which source agencies) and where they are delivered (to which execution and/or sink agencies). Collected documents are included in the document list of the work specification. The initiation agency may also provide documents, together with the information that is required to create the initial work specification.

A source agency which is requested to provide a document can either obtain the document using a non-standardised access method or using the File Transfer, Access and Management (FTAM) standard [15]. Similarly, a sink or execution agency that accepts a document may dispose it in a way that is not standardised or by using the FTAM standard.

A document movement subjob consists of a list of document movement operations. A document movement operation may specify the concatenation of collected documents to form a single document for delivery. It may also specify with a single reference a group of documents, rather than an individual document, which are to be collected from a single source agency.

Figure 3 illustrates the processing of two successive document movement subjobs, one contained in the initial work specification and the other contained in the work specification created after completion of the initial subjob by spawning. The figure shows the submission of a work specification by an initiation agency. This work specification is routed to a source agency to pick up a document, and subsequently forwarded to an execution agency. After processing, the output is sent to a sink agency. Each interaction is performed using work specifications as a device to carry work and information related to that work. 

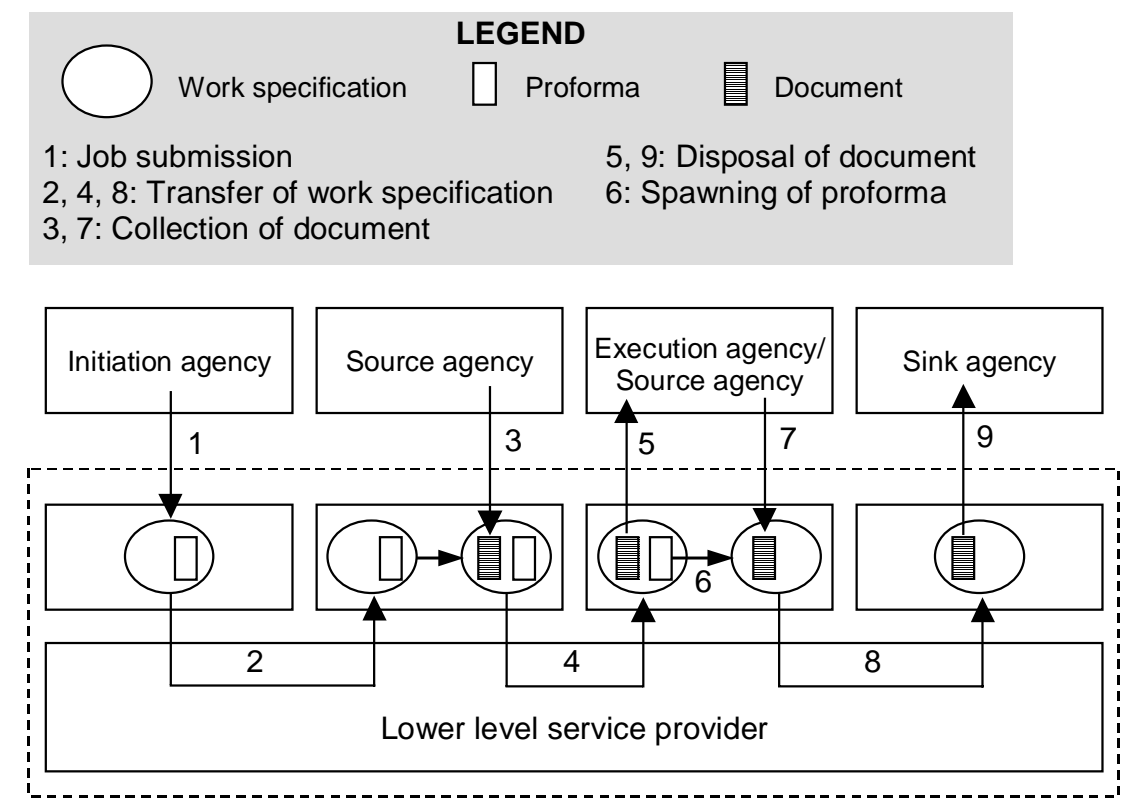

Fig. 3. Example of document movements, accomplished by two successive document movement subjobs.

\subsection{Other JTM subjob types}

There are a number of other JTM subjob types, besides document movement, that are supported by the JTM service provider. Some of these subjob types are briefly discussed in the sequel.

\subsubsection{Report movement}

JTM jobs may take a long time to complete. JTM provides primitives to inquire about the status of a job and introduces the concept of job monitor, which is a system to which reports are sent regarding the progress of a job.

A report movement subjob is contained by a work specification that is created after the occurrence of certain events, such as the normal termination of a subjob, the abnormal termination of a subjob due to the occurrence of errors, the transfer of a work specification and the spawning of a proforma.

A report movement subjob consists of a set of report operations, where each report operation causes a single report to be delivered to a single monitor point (an agency) in a monitor system. Report movement is much simpler than document movement since reports are never collected and route by a report movement subjob, but are always transferred, without intermediate manipulation, from source (the JTM ASE that detected a selected event) to destination (the selected monitor point). 


\subsubsection{Work manipulation}

This subjob type specifies how to modify ongoing work associated with an OSI job. A work manipulation subjob consists of a list of work operations, which cause the target system to display or modify the definition of a work specification for which the system is responsible. There are five operations:

- select, which causes the selection of one or more work specifications. All operations following the selection apply to the selected work specifications until a next selection operation is performed;

- modify, which changes certain fields in a work specification, and thus affects the further processing of the OSI job;

- kill and stop, which cause the agencies in the target system to stop all processing associated with the work specification. The kill operation in addition causes the system to discard the work specification and any results, and;

- display, which causes a copy of parts of the selected work specifications to be placed in a document movement work specification, which is created by spawning the proforma contained in the work manipulation specification.

\subsubsection{Report manipulation}

A report manipulation subjob enables a JTM user to either delete or obtain reports that have been retained by monitor points. This subjob consists of a delete operation or a display operation. A delete operation causes deletion of all received reports about part or all of a specified OSI job. A display operation causes the generation of a display document, constructed from the received reports about the OSI job.

\subsubsection{Transfer manipulation}

A transfer control manipulation subjob consists of a set, check or transfer display operation. These operations apply to a data structure, called transfer control record (TCR). A TCR is used by a JTM ASE to control the transfer of work specifications from one JTM ASE to another.

A set operation causes a new TCR, possibly replacing an existing TCR, to be put into use by a JTM ASE. This operation is used as a kind of filter for work specifications that are awaiting transfer to another JTM ASE. A check operation causes an existing TCR to be checked for correctness by the JTM management centre that originally set the TCR. This operation is used usually after the occurrence of some failure. A transfer display operation causes the generation of a display document, which is constructed from a specified TCR. 


\subsection{Specific areas of concern}

There are a number of areas of concern that apply to JTM activity support, such as transaction support, error handling, security provision and implementation options. These areas are briefly discussed in the sequel.

\subsubsection{Transaction support}

Transaction support means that users can rely on the existence of designated outcomes of a JTM activity. This requires that an initiation agency know which point in the OSI job the service provider can take responsibility for the processing of associated actions as a single atomic action. Three levels of commitment are supported, for instance through the use of the Commitment, Concurrency and Recovery (CCR) standard [18]:

- completion commitment ("on-line" processing): the entire OSI job is completed as an atomic action; all work specifications created as part of the JTM activity have been fully processed, and have ceased to exist.

- agency acceptance commitment: the initial OSI subjob has progressed (at least) to the point at which all documents have been collected from source agencies, and delivered to the sink and/or execution agencies in the target system, where they have been secured for further processing.

- provider acceptance commitment ("off-line" processing): the initial work specification has been created and secured by the service provider and all agencies that will be accessed as part of the initial subjob have been informed.

\subsubsection{Error handling}

A variety of mechanisms are available to cope with the occurrence of errors during the processing of a work specification. For document movement there are three options:

- place an error diagnostic, instead of a requested document, in the document list of the work specification, generate an error diagnostic report, and continue. This action can be taken if a document cannot be obtained from a source agency, but further useful processing is still possible.

- suspend the processing of the work specification for a certain period of time to allow user correction, and generate a non-progress report. This action can be taken if a document cannot be delivered to a sink or execution agency, or when a work specification cannot be transferred to a remote JTM ASE. If the error has not been corrected when the time period expires, then the work specification will be deleted (see below); 
- delete the work specification and generate an abnormal termination report. This action is taken when further useful processing of the work specification is no longer possible and no attempts from users to correct the error are awaited.

In addition to these options, a JTM user may also submit messages with error diagnostics. An message is treated as an event (see report movement) by the local JTM ASE and will generate a report that carries the message.

\subsubsection{Security provision}

Security, in particular authentication, has been recognised as an important requirement for the support of JTM activity. Three mechanisms are available for providing authentication, viz., password protection, third party authentication and audit trace.

Password protection mechanisms are the most common protection mechanism and do not require additional explanation. In the third party authentication mechanisms, the authenticator receives a statement from a trusted party that user identifications present in the communication have already been authenticated. Three levels of trust are recognised, viz., 'unknown'(if there is no other party willing to vouch for the information object being authenticated rather than the object itself), 'known' (the network provides an authenticated network address that can be used to validate the user identity), and 'authenticated' (the user identity is authenticated using encryption techniques). Audit trace mechanisms are used to support the previous mechanism by listing the JTM ASEs that have been involved in the processing of the work specification, and the level of trust associated with them.

Although third party authentication provides a practical method for security provision, the highest level of trust relies on the use of encryption techniques, which are outside the scope of JTM.

\subsubsection{Implementation options}

The JTM standard is functionally rich. In order to provide a flexible approach to implement only a subset of JTM functions, the standard defines two dimensions of functional subsetting.

The first dimension comprises the provision of the essential features of JTM, such that document movement, report movement and work manipulation can be supported, but not report manipulation and transfer manipulation. The second dimension consists of the definition of the functions that JTM ASEs can provide depending on the type of system in which they reside and the 
agency types they support. For example, not all implementations support OSI job submission, document processing or report processing. Any combination of the defined classes of functions constitute a valid JTM implementation.

The JTM protocol relies on a number of other OSI components: CCR, the Association Control Service Element (ACSE), and the Presentation service provider, i.e., the first 6 layers of the OSI protocol stack. In order to increase the acceptance level of JTM, it should be possible to use OSI as well as non-OSI protocols. This can be accomplished when alternative mappings of the JTM protocol on lower level protocol stacks are developed. A possible candidate for such a protocol stack is the TCP/IP protocol suit, enhanced with functions that can support CCR, or another two-phase commitment protocol, and ACSE.

\section{JTM Support of Workflow automation}

In order to establish the conceptual correspondence between workflow and JTM, we enumerate the correspondence between the various concepts and the relationships between those concepts.

An object is an entity with a well-defined boundery and identity with meaning for the problem at hand. An information object is an object that is used in a workflow process to carry the information needed to perform activities. JTM refers to documents, but uses a broad definition for it that renders information objects and documents synonym in practice.

Both in workflow and in JTM, an abstraction is made from the precise nature of an activity, by treating it as a collection of events. An event is something that happens (occurs) at a point in time. An event has no duration. An event in a workflow process corresponds to sending a work item, which is represented in JTM by a work specification.

An actor is any single person, machine or group of actors that trigger events. JTM uses the word agency for this concept. In case of human actors, there is always a process running on a computer that communicates with this person. The word agency refers to this (technological) process, whereas the word actor is used in both (or even combined) ways. At the conceptual level there is no need to distinguish between the two terms, so we use the more neutral word "actor".

An activity is a collection of events that a single actor can trigger in a single span of time. This word is used both in the area of workflow and in JTM. In JTM, there is a work specification that causes the JTM service provider to 


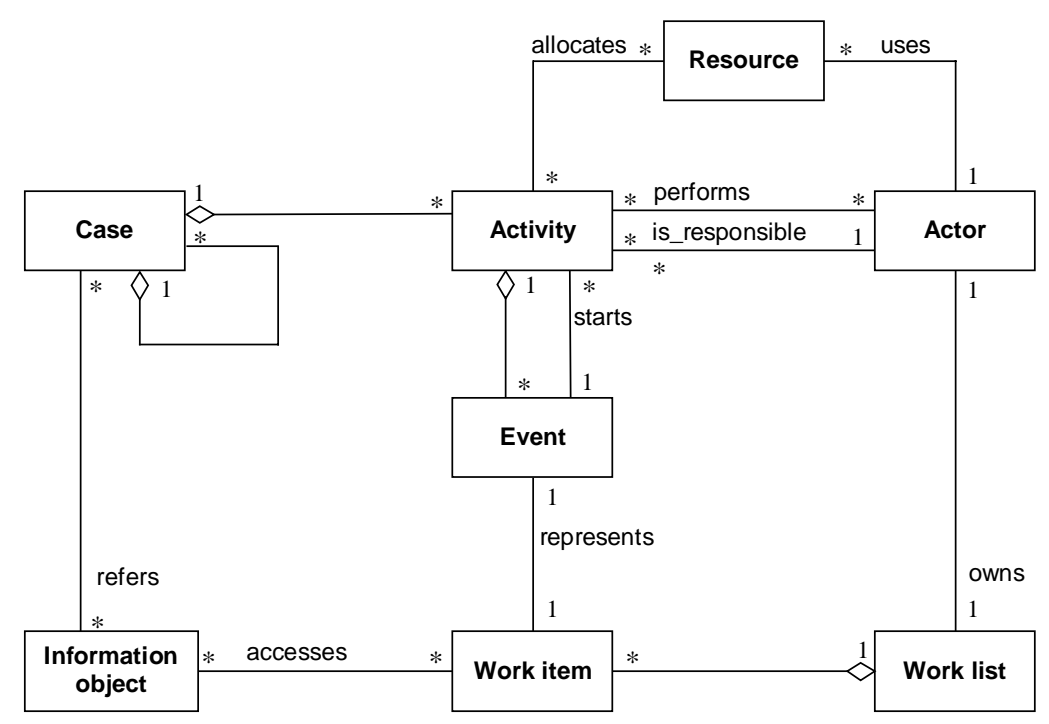

Fig. 4. Conceptual model of a workflow process.

trigger the activity of an appropriate agency. An activity starts as a result of the occurrence of an event, it is performed by an actor and it finishes after all its events have occurred. A difference between events and activities is that an event is instantaneous, while an activity takes time.

A case is a single instance of a process. A case is initiated (synonym: opened) as a result of a particular event (the initial event), processed through the execution of the required activities and completed (synonym:closed) when certain conditions are met. Each case is represented by an information object called case record or case file, in which the actual status of the case is kept. The JTM-equivalent of case is (OSI) job. A job is initiated by an initiation agency and leads to an initial work specification in the JTM service provider. The JTM service provider keeps track of the job while it executes through this work specification (and any of its descendants obtained by spawning).

A resource is something that can be used at any time by a single actor only. The allocation of resources to activities is a competitive coordination (resources are scarce). JTM uses service primitives to involve agencies and other resources to allow for this type of coordination within the workflow process. JTM makes use of the CCR (or equivalent) protocol to provide safe transaction services.

Figure 4 depicts the structure of these concepts as a UML class diagram [5]. In the sequel we describe the relationships between these concepts.

A case may contain several sub-cases. This feature is represented in our model through the use of an aggregation association between a case object class and this class itself. This tree structure hierarchy is suitable to structure and decompose a complex case. A case may also refer to several information objects simultaneously. Besides, different cases may refer to the same information 
object. Transaction mechanisms are provided to ensure safe operations under such circumstances.

Both workflow and JTM consider a case as a set of activities. This feature is represented in our model through the use of an aggregation association between a case object class and an activity object class. An activity consists of a set of events. This feature is represented in our model through the use of an aggregation association between an activity object class and an event object class. In JTM this feature is carried out by a service provider that creates and maintains work specifications. An activity starts some time after the occurrence of the event causing it, but never before. The JTM service provider will do so by detecting the event, after which a service primitive is issued to indicate to the agency that an activity must be started.

Work items in WFMSs correspond to work specifications in the JTM standard. According to JTM, work specifications contain references to the information required to perfom the work. Similarly, in our model a work item accesses an information object. A work item also represents an event. JTM defines the work specification as the physical object that represents an event.

An actor relates to an activity in two different ways. An actor either performs an activity or $i s$ responsible for an activity. The difference between these types of relationship is the following: an actor responsible for an activity, actually may not be one who will perform this activity. But if there is something wrong with this activity the actor responsible for it must be contacted. Besides, there must be only one actor responsible for an activity while there may be several actors performing this activity. The completion of an activity is signalled by the JTM service provider, which takes the appropriate action according to the work specification at hand. JTM does not have any specific provisions for properly organising the responsibilities because this feature is a matter of the application built on top of JTM. The regular JTM service primitives can be used to put responsible actors to work when certain conditions are not met, quite similar to the way in which other actors get work requests when these conditions are met.

An actor also owns a work list. A work list consists of a set of work items associated with an actor. All actors who work on a case or on any of its subcases can inspect or change any information object in that case by means of its work list.

An activity allocates resources, which are used by the actors while performing an activity. In JTM, this is either a local matter or it is requested via the JTM service or through another service outside the scope of JTM. Transaction mechanisms are provided to safeguard the claims and releases of resources. 


\section{Discussion}

The JTM standard is useful to support workflow tools, because the workflow concepts of case, activity, actor, event, object, and resource can be represented by the JTM concepts of OSI job, activity, agency, work specification, document and resource, respectively. Furthermore, the relationships between those concepts can be represented by similar relationships or service primitives in JTM. That is sufficient evidence to show that JTM is conceptually suitable to support workflow processes, which justifies a positive answer to our research question. In answering this question, we have used only deductive reasoning (as opposed to inductive reasoning). This allows us to claim general validity of the result. Of course, practical aspects require further experiments, since we have established conceptual correspondence only. Such experiments are considered beyond the scope of this paper.

A WFMS can be considered a useful application of JTM because it draws on most (if not all) of JTM's functionalities. Apart from triggering activities by means of communicating work specifications between JTM ASE's, business processes require the use of monitors for reporting purposes, transaction control for distributed atomicity and consistency of activities, and security provisions. All of these things are provided by JTM.

This result affects the current efforts of the WfMC, which is currently engaged in the process of writing API specifications for the different interfaces defined in the Coalition's reference model, such as [35]. These efforts can be complemented by taking the JTM service specification [16] and aligning both. This renders the design of a workflow protocol obsolete because the JTM protocol [17] may be reused. The choice to do so must be taken by the Coalition, of course. The fact that the JTM standard is not proprietary to any vendor helps to make it politically acceptable in a vendor dominated community as the WfMC. The fact that JTM is an ISO standard is helpful to convince Coalition members of the quality of JTM, because ISO has strict (and long and labour intensive) procedures to ensure that a standard is sound before being approved.

Currently workflow management systems have predominantly client/server architectures [28], in which knowledge about the processes is centralised. The further development of current workflow management systems from a single server architecture to multi server architecture is anticipated, e.g. [30]. Remarkably enough, this development was anticipated by the JTM design team as well, as early as 1989. Workflow applications are inherently distributed, because business processes in general are distributed over different organisational units inside and outside an organisation. Although a centralised architecture simplifies the design of functionalities, such as synchronisation, monitoring and 
transaction support, a distributed architecture is an acknowledged requirement for the next generation of WFMSs [3,30]. Distributed servers provide several advantages, such as a greater scalability, an increased configuration flexibility and an increased resilience to failures. The architecture of the JTM standard assumes that JTM application service elements are distributed. The workflow server functions are typically performed by the JTM ASE, which communicates with the initiation agency. This means that the server performance burden is distributed over the ASE's that serve the various initiation agencies. In this context, the integration of JTM and Internet developments should be further investigated.

An interesting question to discuss at this point is why workflow and JTM have not been related to one another earlier. We can but speculate on the answer, especially since the conceptual correspondence between the two is so apparent. We think it is caused by a combination of factors. Time plays a role, because the notion of workflow has been used widely only during the past few years. JTM being short of good applications is a factor too, because it never became a widely known standard. Finally, we think that there should be more interest from both fields in each other's developments.

\section{Conclusion}

We contend that interoperability among different brands of WFMSs has not been achieved so far. In addition, these efforts seem to be isolated from other standardisation activities, notably those of ISO and ITU-TS (formerly CCITT) in the area of Open Systems Interconnection (OSI).

We have shown that the OSI JTM standard can be considered as a suitable workflow standard for remote interworking. This standard provides a rich functionality, which includes document movement, report movement and work manipulation. Furthermore, it supports a number of important features, such as transactions, error handling and security provision. Although JTM is a complex standard, useful subsets are defined, which limit implementation complexity and costs per end-system.

An area of future work is to align the current efforts of the WfMC with the existing documentation of the JTM standards. By doing so, much work is avoided and further development of WFMS is achieved faster. Another area of future work is the provision of alternative mappings of the JTM protocol, such that non-OSI protocol stacks can be used instead of the OSI protocol stack to provide the lower level communication support. 


\section{Acknowledgements}

We wish to thank Mark de Weger and Duane Truex for their useful comments on earlier versions of this paper. Cléver Ricardo Guareis de Farias is supported by CNPq - Brazil.

\section{References}

[1] K.R. Abbott and S.K. Sarin, Experiences with workflow management: issues for the next generation, Proceeding of the ACM 1994 Conference on Computer Supported Cooperative Work (CSCW'94) (1994) 113-120.

[2] A. Agostini, G. De Michelis and M.A. Grasso, Rethinking CSCW Systems: The Architecture of Milano, Proceedings of the Fifth European Conference on Computer Supported Cooperative Work (ECSCW'97) (1997) 33-48.

[3] G. Alonso and C. Mohan, WFMS: The Next Generation of Distributed Processing Tools, Jajodia, S. and Kerschberg, L. (Eds.), Advanced Transaction Models and Architectures., (Kluwer Academic Publishers, 1997) 35-62.

[4] D. Barbara, S. Mehrota and M. Rusinkiewicz, INCAS: Managing Dynamic Workflows in Distributed Environment, Journal of Database Management Vol. 7 no. 1 (IDEA Group Publishing, 1996) 5-15.

[5] G. Booch, J. Rumbaugh and I. Jacobson, Unified Modeling Language User Guide (Addison-Wesley, 1998).

[6] G. Bracchi and B. Percini, The design requirements of office systems, ACM Transactions on Office Systems Vol. 2 no. 2 (1984) 151-170.

[7] C.A. Ellis, Information control nets: A mathematical model of office information systems, Proceedings of the ACM-SIGOA Conference on Simulation, Measurement and Modeling of Computer Systems (1979) 225-239.

[8] C.A. Ellis and M. Bernal, OFFICETALK-D: An Experimental Office Information System, Proceedings of the ACM-SIGOA Conference on Office Automation Systems (1982) 131-140.

[9] M. Felhoffer, Communication support for distributed applications (Elsevier, 1992) 215-228.

[10] D. Georgakopoulos, M. Hornick and A. Sheth, An Overview of Workflow Management: From Process Modeling to Workflow Automation Infrastructure, Distributed and Parallel Databases Vol. 3 no. 2 (1995) 119-153.

[11] A. Geppert and D. Tombros, Event-based Distributed Workflow Execution with EVE, Proc. IFIP International Conference on Distributed Systems Platforms and Open Distributed Processing (Middleware '98), The Lake District (England) (1998) 427-442. 
[12] A. Grasso, J. Meunier, D. Pagani and R. Pareschi, Distributed Coordination and Workflow on the World Wide Web, Computer Supported Cooperative Work: The Journal of Collaborative Computing Vol. 6 no. 2/3 (1997) 175-200.

[13] D. Hollingsworth, The Workflow Reference Model, WFMC-TC-1003, Issue 1.1 (1994). Available at http://www.aiim.org/wfmc/files/refmodel/rmv1-16.pdf.

[14] M. Hsu and C. Kleissner, ObjectFlow: Towards a Process Management Infrastructure, Distributed and Parallel Databases Vol. 4 no. 2 (1996) 169194.

[15] ISO, Information Processing Systems - Open Systems Interconnection File Transfer, Access and Management, Service Definition and Protocol Specification, International Standard 8571-3 and 8571-4 (ISO, 1988).

[16] ISO, Information Processing Systems - Open Systems Interconnection - Job Transfer and Manipulation Concepts and Services, International Standard 8831 (ISO, 1989).

[17] ISO, Information Processing Systems - Open Systems Interconnection Specification of the Basic Class Protocol for Job Transfer and Manipulation, International Standard 8832 (ISO, 1989).

[18] ISO, Information Processing Systems - Open Systems Interconnection Commitment, Concurrency and Recovery Service Element, Service Definition and Protocol Specification, International Standard 9804 and 9805 (ISO, 1990).

[19] S. Joosten, G. Aussems, M. Duitshof, R. Huffmeijer and E. Mulder, WA-12: an empirical study about the practice of workflow management, University of Twente, Enschede, The Netherlands (Research monograph, ISBN 90-365-0683$2,1994)$.

[20] K. Kumar and H. van Dissel, Conflicts and cooperation in interorganizational information systems, Proceedings of the European Conference on Information Systems (ECIS'95) (1995).

[21] C. L'Anson and A. Pell, Understanding OSI Applications (PTR Prentice Hall, 1993).

[22] H. Lavana, A. Khetawat and F. Brglez, Internet-based workflows: a paradigm for dynamically reconfigurable desktop environments, Proceedings of the International ACM SIGGROUP Conference on Supporting group work: the integration challenge (GROUP '97), Phoenix (USA) (1997) 204-213.

[23] F. Leymann and W. Altenhuber, Managing business processes as an information resource, IBM Systems Journal Vol. 33 no. 2 (1994) 326-348.

[24] D.R. McCarthy and S.K. Sarin, Workflow and Transactions in InConcert, IEEE Data Engineering Bulletin Vol. 16 no. 2 (1993) 53-56.

[25] R. Medina-Mora, T. Winograd, R. Flores and F. Flores, The action workflow approach to workflow management technology, Proceedings of the ACM 1992 Conference on Computer Supported Cooperative Work (CSCW'92) (1992) 281288. 
[26] J. Miller, A. Sheth, K. Kochut and X. Wang, CORBA-Based Run-Time Architectures for Workflow Management Systems, Journal of Database Management (Special Issue on Multidatabases) Vol. 7 no. 1 (1996) 16-27.

[27] J. Miller, D. Palaniswami, A. Sheth, K. Kochut and H. Singh, WebWork: METEOR's Web-based Workflow Management System, Journal of Intelligent Information Systems Vol. 10 no. 2 (1998) 1-30.

[28] C. Mohan, State of the Art in Workflow Management System Research and Products, Tutorial presented at: NATO Advanced Study Institute (ASI) on Workflow Management Systems and Interoperability, Istanbul, August/1997.

[29] C. Mohan, Recent Trends in Workflow Management Products, Standards and Research, Proc. NATO Advanced Study Institute (ASI) on Workflow Management Systems and Interoperability, Istanbul, August/1997 (Springer Verlag, 1998).

[30] C. Mohan, G. Alonso, R. Guenthoer and M. Kamath, Exotica: A Research Perspective on Workflow Management Systems, Data Engineering Bulletin (Special Issue on Infrastructure for Business Process Management) Vol. 18 no. 1 (1995) 19-26.

[31] S. Sarin, K. Abbott and D. McCarthy, A process model and system for supporting collaborative work, Proceedings of the ACM-SIGOIS Conference on Organizational Computing Systems (1991) 213-224.

[32] K.D. Swenson, R.J. Maxwell, T. Matsumoto, B. Saghari and K. Irwin, A Business Process Environment Supporting Collaborative Planning, Collaborative Computing Vol. 1 no. 1 (1994) 15-34.

[33] J. Webster, Network of Collaboration or Conflict? The Development of EDI, Proceedings of the Sixth International Conference Electronic Data Interchange and Interorganizational Systems (1993) 149-170.

[34] Workflow Management Coalition, Terminology \& Glossary, (Workflow Management Coalition, WFMC-TC-1011, Issue 2.0, 1996). Available at http://www.aiim.org/wfmc/files/glossary/glossary.doc.

[35] Workflow Management Coalition, Workflow Client Application (Interface 2): Application Programming Interface (WAPI) Specification, (Workflow Management Coalition, WFMC-TC-1009, Version 2.0e (Beta), 1997). Available at http://www.aiim.org/wfmc/docs/IF2V20e.PDF.

[36] Workflow Management Coalition, Workflow and the Internet: Catalysts for Radical Change, (Workflow Management Coalition White Paper, 1998). Available at http://www.aiim.org/wfmc/pr/finalwp.pdf. 